Thorax (1973), 28, 627.

\title{
Massive aortic incompetence associated with the Björk-Shiley prosthesis
}

\author{
GEOFFREY H. SMITH and KRISHNA CHANDRA \\ Sheffield Regional Cardio-Thoracic Unit, Northern General Hospital, Sheffield S5 7AU
}

\begin{abstract}
Smith, G. H., and Chandra, K. (1973). Thorax, 28, 627-630. Massive aortic incompetence associated with the Björk-Shiley prosthesis. Three patients are described. In each, a diseased aortic valve was replaced by a Björk-Shiley valve prosthesis. For different reasons, ventricular fibrillation occurred when the patient was still on cardiopulmonary bypass. In these circumstances, a massive leak occurred through the valve and caused overdistension of the left ventricle.

Subsequent examination of the valves in the laboratory showed that, under the conditions described above, there was a leakage equal in volume to half the forward flow.
\end{abstract}

The continual search for an improved cardiac valve prosthesis has recently concentrated on the development of central flow disc valves. Björk described such a valve in 1969 and in subsequent reports has commented on its behaviour in the aortic and in the mitral area (Björk and Olin, 1970a and $b$; Björk, 1970). Other workers have used the valve extensively.

Up to November 1972, 110 patients have had a total of 130 Björk-Shiley valves inserted at this surgical unit. Sixty patients have undergone aortic valve replacement and in 21 of these the mitral or the tricuspid valve was also replaced.

On three occasions recently, the insertion of a Björk-Shiley valve into the aortic area has led to severe valvular leakage, occurring during cardiopulmonary bypass. The purpose of this paper is to describe these cases and the results of laboratory testing of the valves.

\section{CASE HISTORIES}

CASE 1 A man aged 44 years complained of angina pectoris and dyspnoea on effort for five years. Examination revealed a fit man. The pulse was anacrotic in character and the arterial pressure $170 / 100 \mathrm{mmHg}$. Signs of left ventricular overactivity were present. A marked systolic and early diastolic murmur were heard in the aortic area.

Chest radiography showed a large heart with a prominent ascending aorta. Calcification was visible in the aortic valve. The electrocardiogram revealed sinus rhythm, left axis deviation $\left(-45^{\circ}\right)$, and left ventricular hypertrophy.

On cardiac catheterization it proved impossible to pass the catheter across the aortic valve. Aortography showed marked aortic regurgitation into an efficiently contracting left ventricle. Coronary arteriography was performed, showing complete proximal obstruction in the anterior descending coronary artery.

At operation the external appearances of the heart were typical of isolated aortic stenosis and regurgitation. Cardiopulmonary bypass was begun using a nonblood prime of Hartmann's solution and plasma. The arterial return was inserted into the ascending aorta. The aortic valve was found to be grossly calcified and stenotic. Coronary perfusion was established and the heart remained in sinus rhythm. The valve was excised and replaced with a $25 \mathrm{~mm}$ Björk-Shiley aortic valve with a Delrin disc. The aorta was repaired and bypass was stopped. The cardiac action, however, deteriorated over a five-minute period and the decision was made to perform coronary artery bypass grafting.

The left ventricle was vented at its apex and the heart was electrically fibrillated. A segment of saphenous vein was easily inserted into the middle onethird of the anterior descending coronary artery. During the period of ventricular fibrillation, however, the amount of blood draining from the left ventricular vent increased from $300 \mathrm{ml} / \mathrm{min}$ to $3,000 \mathrm{ml} / \mathrm{min}$ over a very short period and the amount suddenly became more than the volume with which the vent could deal. Sudden gross left ventricular distension occurred. The aorta was cross-clamped and re-opened. The prosthesis was inspected and appeared to be sewn into position satisfactorily. Coronary perfusion was established via the coronary arteries and saphenous vein, but the heart remained dilated and atonic with a slow inefficient contraction.

At necropsy the prosthesis was found to be securely sewn in the subcoronary position. The saphenous vein graft was also seen to be satisfactorily positioned. The entire thickness of the myocardium of the left ventricle 
was occupied by petechial haemorrhages and it was concluded that the left ventricle had been unduly distended by the severe aortic reflux.

The valve was returned to the laboratory and tested in the manner described below. At a steady flow of $51 . / \mathrm{min}$ at a pressure of $60 \mathrm{mmHg}$ the flow through the valve, between the ring and the disc, was $2,400 \mathrm{ml} / \mathrm{min}$ although the disc was not moving and was apparently well positioned within the ring of the prosthesis.

CASE 2 A 52-year-old man presented with a 12-year history of dyspnoea on exertion, tiredness, and chest pain. The dyspnoea was severe and the chest pain occurred on the slightest exertion.

Physical examination revealed a thin man with a malar flush. The pulse was irregular and collapsing. The arterial blood pressure was $120 / 60 \mathrm{mmHg}$. Auscultation of the heart revealed the murmurs of mitral regurgitation and aortic stenosis with regurgitation. Chest radiography showed a large heart with calcification in the aortic and mitral valves. Electrocardiography showed atrial fibrillation and biventricular hypertrophy.

Cardiac catheterization was performed and showed evidence of aortic stenosis and regurgitation, mitral regurgitation, and moderate elevation of pulmonary vascular resistance (ca 4 units). Mitral and aortic valve replacement was advised.

At operation the external appearances of the heart were consistent with the presence of aortic and mitral valve regurgitation. The myocardium seemed irritable, however, and multiple ventricular ectopic beats occurred. Cardiopulmonary bypass was begun using a non-blood prime and with the return to the ascending aorta. The heart fibrillated as soon as the left ventricular vent was inserted but returned to sinus rhythm when coronary perfusion had been established. The mitral and aortic valves were found to be calcified and incompetent. They were excised and replaced with Björk-Shiley valves with Delrin discs. The cardiac incisions were closed and the air was evacuated from the heart. As the left ventricular vent was being removed, however, ventricular fibrillation again occurred and gross distension of the left ventricle occurred in spite of the vent functioning properly. In addition the arterial pressure dropped from 65 to $25 \mathrm{mmHg}$. Using a small probe inserted through the aortic incision, the disc of the aortic prosthesis was pushed into position and prevented from oscillating. The reflux into the left ventricle persisted unabated and therefore the aorta was clamped and reopened and coronary perfusion begun. The prosthesis was inspected and appeared to be securely in position. It was excised and replaced with a composite seat StarrEdwards value. After this heart proved difficult to convert back to an effective rhythm, but eventually the patient managed to support a fair circulation with the aid of an intravenous infusion of adrenaline. The cardiac action progressively deteriorated over the next 24 hours.

At necropsy, the wall of the left ventricle was found to be the site of many small haemorrhages and oedema. Death was due to left ventricular failure, the cause of which was consistent with an acute mechanical overdistension.

The aortic valve was not examined in our laboratory on this occasion but was returned to Shiley Laboratories. The valve was examined by them and reported to be well within the limits of tolerance of the manufacturer.

CASE 3 A man aged 47 years had been known to have a cardiac murmur for 41 years. Symptoms of dyspnoea and tiredness had been present for five years and more recently several attacks of syncope had occurred.

Physical examination showed a man of normal physique, anacrotic pulse, and a murmur of severe aortic stenosis. Chest radiography showed a normally sized heart but dilatation of the ascending aorta. Electrocardiography revealed sinus rhythm and left ventricular hypertrophy.

Cardiac catheterization showed calcification of the aortic valve and severe stenosis. Some aortic regurgitation was demonstrated.

At operation the ascending aorta was found to be moderately dilated but very thin-walled. Cardiopulmonary bypass was begun using a non-blood prime and with the arterial return to the ascending aorta. Coronary perfusion was established and the calcified aortic valve removed. Extreme thinness of the aorta was noted to continue into the subvalvar region. The valve was replaced with a $25 \mathrm{~mm}$ Björk-Shiley valve with a Pyrolite disc. The closure of the aorta was extremely difficult and although the heart took over the circulation well, there was bleeding from the left side of the suture line as the arterial pressure rose. Accordingly, bypass was re-established and the aorta was cross-clamped after the left ventricle had been vented. The aorta was reopened and a tear was found, extending from the left-hand side of the aortotomy into the posterior aspect of the aorta just above the valve ring. This was rapidly repaired using buttressed sutures and the aortotomy was again repaired. Ventricular fibrillation occurred as the coronary perfusion was re-established and the return from the left ventricular vent rapidly increased from $300 \mathrm{ml} / \mathrm{min}$ to $3,500 \mathrm{ml} / \mathrm{min}$, and left ventricular distension occurred. The aorta was cross-clamped again in order to prevent this excessive reflux and the aortotomy was opened for a third time. The intention was to replace the prosthesis but the tear in the back of the aorta was found to have extended down into the left ventricular outflow and this proved impossible to repair.

At necropsy, the valve had been sewn into position satisfactorily.

LABORATORY TESTING The aortic valve prostheses were removed from each patient at necropsy. The valve from case 2 was sent to Shiley Laboratories for examination.

The other two valves, one with a Delrin disc and the other with a Pyrolite disc, were tested in such a way as to attempt to reproduce the conditions found during cardiopulmonary bypass with a fibrillating heart (Figure). 


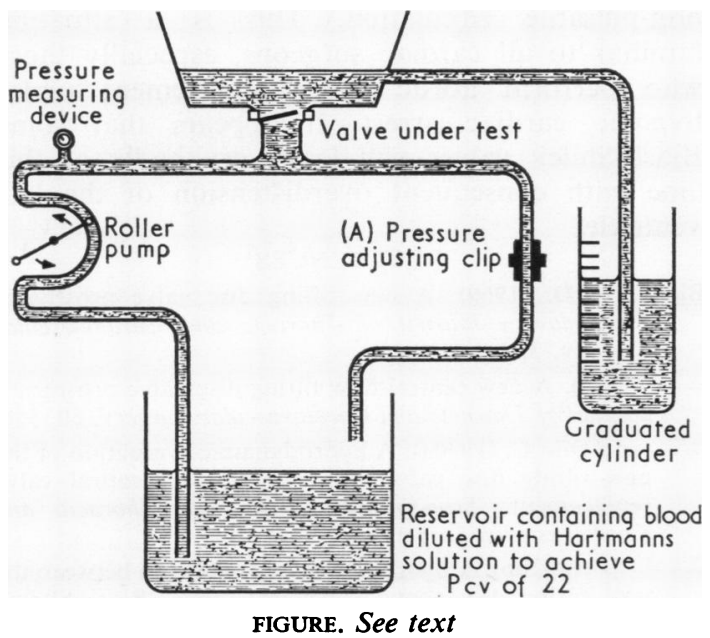

The perfusate was blood diluted with Hartmann's solution to give a haematocrit of $22 \%$. The pump output was 5 litres per minute and the pressure was adjusted to $60 \mathrm{mmHg}$ by means of the screw clip (A). The behaviour of each valve was recorded by slow motion cinephotography (64 frames/sec). The gap between the disc and the ring was measured by a micrometer in each valve.

\section{RESULTS}

Shiley Laboratories report that the valve submitted to them conformed to the limits of tolerance. These limits were, however, not stated.

The results of laboratory testing on the artificial circuit are shown in the Table.

T A B L E

\section{RESULTS OF LABORATORY TESTING}

Standard conditions-5 litres flow around circuit at a pressure of $60 \mathrm{mmHg}$ of a mixture of blood and Hartmann's solution with PCV of 22

\begin{tabular}{l|c}
\hline \multicolumn{1}{c|}{ Valve } & $\begin{array}{r}\text { Regurgitant } \\
\text { Flow } \\
\text { (ml/min) }\end{array}$ \\
\hline $\begin{array}{l}\text { Starr-Edwards 10A 2310 } \\
\text { Björk-Shiley with Delrin disc (case 1) } \\
\text { Björk-Shiley with Pyrolite disc } \\
\text { Randomly selected Björk-Shiley with Pyrolite disc }\end{array}$ & 300 \\
& 2,400 \\
3,000 \\
300
\end{tabular}

In the valve from case 1 the regurgitant flow was $2,400 \mathrm{ml} / \mathrm{min}$ under the standard conditions.

In the valve from case 3 the leak was 3 litres per minute. The leak through a randomly selected unused Björk-Shiley valve with a Pyrolite disc was $300 \mathrm{ml} /$ minute. A similar small leak occurred through a Starr-Edwards 2320 10A valve inserted into the housing.

Slow motion cinephotography showed no visible movement of the disc in the prosthesis from case 1 . The disc in the valve from case 2 showed a rapid oscillation which ceased at a pressure of $250 \mathrm{mmHg}$. At this pressure the amount of regurgitant flow through the valve dropped to about $300 \mathrm{ml} /$ minute.

The clearance between the disc and ring measured $0.0625 \mathrm{~mm}$ in the valve from case 1 (Delrin disc) and $0.063 \mathrm{~mm}$ in the valve from case 3 (Pyrolite disc).

\section{DISCUSSION}

The central-flow tilting disc valve prosthesis was designed by Björk and has been available in this country since 1971 .

Two types of valve are available. The prosthesis designed for insertion into the aortic area has a disc opening to an angle of $60^{\circ}$ and that for the mitral area has a disc opening to $50^{\circ}$.

The advantages claimed for this type of valve are three. First, the central orifice of the valve is large in comparison with the external diameter. This results in low pressure gradients across the valve during high cardiac outputs. Secondly, the disc moves easily, having a low inertia, and does not impact against the valve ring. This is said to reduce haemolysis but also allows a degree of regurgitation said to be about $10 \%$ of the stroke volume. Finally, the valve has a low profile and is particularly suitable for mitral valve replacement associated with a small left ventricle.

When first available the disc was made of Delrin ${ }^{1}$. This material is a durable plastic. Unfortunately, it does tend to swell by the absorption of fluid and this fact demands that the clearance between the disc and the valve ring is quite large.

Recently the disc has been available in a compressed silica carbon alloy form, Pyrolite ${ }^{2}$. This disc does not swell during sterilization, and consequently the gap between the disc and the ring is said to have been reduced to $0.0254 \mathrm{~mm}(0.001 \mathrm{in})$ on average and is said to be made to much closer tolerances.

Our measurements of disc/ring clearance in the valves have shown that the distance may be increased beyond the level claimed. Difficulty arises in explaining how such a large degree of leak occurred in valve 1 . Possibly a phenomenon similar to that observed in the valve from case 3 was present-namely oscillation of the disc but at a much higher frequency. If this is so, then it is difficult to explain why the regurgitation occurring in case 2 persisted in spite of the disc being forcibly held in the closed position.

Few complications have been reported resulting 1E. I. Du Pont Nemours and Co. Inc., Wilmington, Del., U.S.A. 2Gulf General Atomic Inc., San Diego, California, U.S.A. 
from the use of the Björk-Shiley prosthesis to replace the aortic valve. Cokkinos et al. (1971) have reported a case in which massive thrombosis occurred around the prosthesis in spite of the patient receiving oral anticoagulant therapy. Sixty Björk-Shiley valve prostheses have been inserted in this unit. Problems related to the valve have occurred only on the occasions described above. The massive leakage occurs with only a minority of valves and only in the presence of ventricular fibrillation and a non-pulsatile artificial circulation. Given this combination of circumstances, however, the degree of leakage is so severe as to impair the perfusion of the tissues and to cause left ventricular damage by overdistension. This latter feature may significantly affect the outcome of the operation.

The conditions under which the valves were tested were highly artificial and do not mimic in any way the physiology of a normal circulation supported by a beating heart. Nevertheless the objective of the laboratory test was to reproduce the circumstances found during operation, that is, a heart in ventricular fibrillation and an artificial non-pulsatile circulation. This is a situation familiar to all cardiac surgeons, especially those who perform aortic valve replacement under hypoxic cardiac arrest. It appears that some Björk-Shiley valves will leak very badly at this time with consequent overdistension of the left ventricle.

\section{REFERENCES}

Björk, V. O. (1969). A new tilting disc valve prosthesis. Scandinavian Journal of Thoracic and Cardiovascular Surgery, 3, 1.

- (1970). A new central-flow tilting disc valve prosthesis. Journal of Thoracic and Cardiovascular Surgery, 60, 355.

- and Olin C. (1970a). A hydrodynamic evaluation of the new tilting disc valve (Björk-Shiley) for mitral valve replacement. Scandinavian Journal of Thoracic and Cardiovascular Surgery, 4, 37.

(1970b). A hydrodynamic comparison between the new tilting disc aortic valve prosthesis (Björk-Shiley) and the corresponding prostheses of Starr-Edwards, Kay-Shiley, Smeloff-Cutter and Wada-Cutter in the pulse duplicator. Scandinavian Journal of Thoracic and Cardiovascular Surgery, 4, 31.

Cokkinos, D. V., Voridis, E., Bakoulas, G., Theodossiou, A., and Skalkeas, G. D. (1971). Thrombosis of two highflow prosthetic valves. Journal of Thoracic and Cardiovascular Surgery, 62, 947. 